\title{
White Light Interference Microscopy System Design
}

\author{
Sébastien Marbach*a ${ }^{*}$ Christophe Cordier ${ }^{\mathrm{a}}$, Rémy Claveau ${ }^{\mathrm{b}}$, Thierry Engel ${ }^{\mathrm{a}}$, Paul Montgomery ${ }^{\mathrm{a}}$, \\ Manuel Flury*a \\ ${ }^{a}$ ICube, University of Strasbourg - CNRS - INSA, 300 Boulevard Sébastien Brand, F-67412 \\ ILLKIRCH, France; ${ }^{b}$ Dept. of Computer Science, University College London, Malet Place, London \\ WC1E 7JG, United Kingdom
}

\begin{abstract}
Interference microscopy is a non-destructive full-field imaging method, mainly used to measure the surface topography of different samples. In this work, two designs for improving the signal quality are described. The first consists of an original vertically orientated breadboard interferometer, in a Linnik configuration. The mechanical design of the arms allows the independent control and alignment of the coherence and the focal plane positions for optimizing fringe contrast. A low noise 16-bit camera is used to improve the sensitivity. The second interferometer is based on a Thorlabs tube system, with a Nikon Mirau Objective and a white LED, all controlled with IGOR Pro software or Labview, with the aim of being more compact, flexible and mobile. For both systems, an evaluation of the interferometric signal quality is performed, whereas the difference in lateral resolution by considering the $3 \mathrm{D}$ nature of the interferometric system, or only its $2 \mathrm{D}$ imaging abilities, is explored.
\end{abstract}

Keywords: White Light Interferometry, Köhler Illumination, Linnik, Mirau, Phase Shifting Interferometry, Coherence Scanning Interferometry, Resolution Limit, Optical Metrology

\section{INTRODUCTION}

The characterization of surface topography is often performed with techniques such as stylus profilometry, confocal microscopy, and AFM for small nm-structures ${ }^{1-3}$. However, if the sample topography needs to be measured at the micrometer scale laterally, white light interference microscopy is often preferred ${ }^{4-6}$ since it presents the advantages of being nondestructive, cost-effective, easy to use, fast and allowing full-field imaging with a nanometric sensitivity along the optical axis. The lateral resolution can be further improved by combining this method with microspheres ${ }^{7-10}$. If the sample does not present step heights larger than half of the central illumination wavelength (with a microscope working in reflection), phase shifting microscopy (PSM) can be performed to obtain the topography, based on quantitative phase determination ${ }^{11}$. With steps greater than half of the wavelength, it becomes necessary to take advantage of the short coherence length of the source. Since the fringe intensity is maximum only when the optical path difference between the arms is null, it is possible to use these white-light fringes as an optical probe. By making a scan over the sample roughness height and topography can be thus obtained. This method is commonly known as White Light Scanning Interferometry (WLSI), or Coherence Scanning Interferometry (CSI) ${ }^{12-14}$. White light interferometry can also be used to perform characterization over the depth when the sample is transparent ${ }^{15,16}$, a method often used in biology, under the name of Full-Field Optical Coherence Tomography (FF-OCT) or Full-Field Optical Coherence Microscopy (FF$\mathrm{OCM})^{17,18}$, for instance to provide photothermal ${ }^{19}$ or elastographic ${ }^{20}$ information, or to detect skin cancer ${ }^{21,22}$.

In this paper, two original designs of white light interferometer (WLI) working in the reflective mode are presented. The first system is an innovative vertically orientated breadboard interferometer in a Linnik configuration. This has been designed to meet the need for a high-quality interferometric signal, not through signal processing but with hardware improvements such as the use of high quality objectives, an accurate piezo-system and a low noise camera. The design process, from computer design until the achievement of the setup is explained. The choices were made with the aim of reducing noise sources as much as possible to enhance the fringe signal. Just enough degrees of freedom were used to allow the adjustment of certain key parameters without making overly complicated alignment of the interferometer.

*marbachs@ unistra.fr; phone +33 3688546 14; mflury@unistra.fr, phone + 333881447 47; http://icubeipp.unistra.fr/en/index.php/Home 
The second WLI is based on a Thorlabs tube system in the Mirau, configuration. The purpose of this setup is to be low cost, more compact and to make faster measurements. The performance of these devices is illustrated through topographic measurement and with the lateral resolution. Indeed, since interferometric system supplies results in $3 \mathrm{D}$, it is not sufficient to describe the lateral resolution of the system based only on its 2D imaging abilities, with the classical Abbe or Rayleigh criteria. Usually, the lateral resolution that these systems can achieve, while providing relevant information on the measured height, is worse than the 2D lateral resolution ${ }^{23-25}$. This is why Leach et al. ${ }^{23}$, De Groot et $a l .^{24}$, and Lehmann et $a .^{25}$, propose to take into consideration the global 3D optical transfer function of the interferometer, to define the lateral resolution. A criterion of lateral resolution that depends on the height measured is exploited to highlight this aspect.

\section{WHITE LIGHT INTERFERENCE MICROSCOPY PROPERTIES}

White Light Interference Microscopy consists in acquiring successive interferometric images, with the introduction of a known optical path difference (OPD) between each image. Experimentally, the OPD is modified by adjusting the distance travelled by the light between the objective and the sample, thanks to a piezoelectric system mounted on the objective or under the sample. The piezo translator is moved step by step along the optical axis $\mathrm{z}$ to image the entire sample surface with the fringe pattern. At each step, an XY interferometric image is acquired and stored in an XYZ stack of interferometric images, as illustrated in Fig 1(a). Then, pixel by pixel the interferometric signal along the depth $\mathrm{z}$ is extracted. At a given pixel $(x, y)$ from the XYZ stack, the intensity I measured as a function of the depth $\mathrm{z}$ can be represented as in Fig 1(b). Since the maximum of the fringe signal corresponds to a null OPD, this fringe can be used as a reference. With the position of the piezo system being determined with nanometer sensitivity, determining the position of the fringe maximum in the image, allows the determination of the topography of the sample. To be more robust, the position of the piezo can be determined at the maximum of the fringe envelope.

(a)

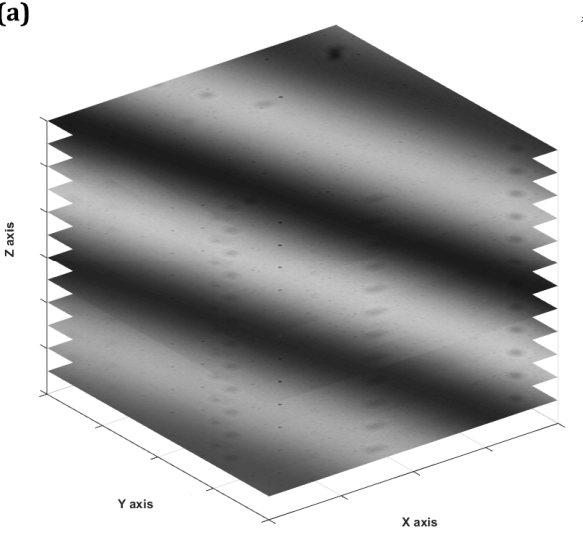

(b)

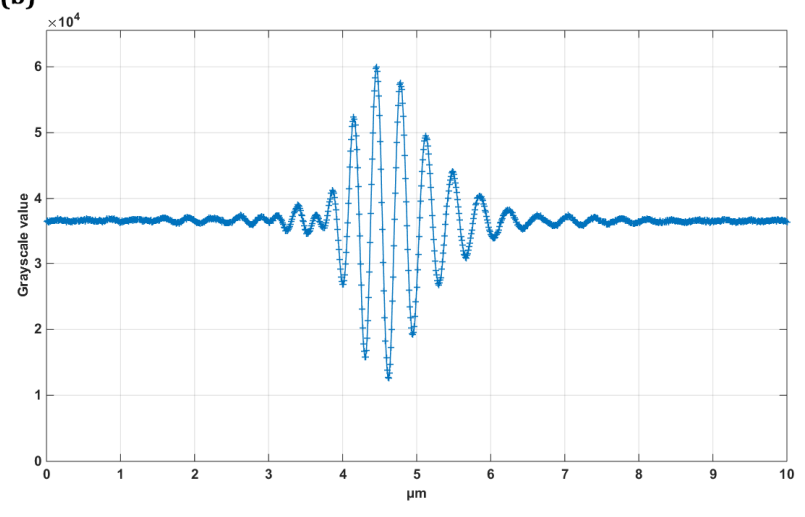

Figure 1. (a) XYZ stack of interferometric images, (b) Interferometric intensity extracted in one pixel (x,y) along the optical axis $\mathrm{z}$.

In this case, the piezo step should be small enough to correctly sample the interferometric signal and so be able to detect with accuracy the maximum of the fringe envelope. The microscope objective has a given Numerical Aperture (NA) so that the reflected light can be collected with an angle $\theta$ up to the maximum angle $\theta_{\max }$ accepted by the objective, and defined in air as the arcsinus from the NA. Beams coming back from the reference arm and the object arm with an angle $\theta$ between them can interfere together. So all the waves included in the cone of light with an apex angle of $2 \cdot \theta_{\max }$, must be considered in the interferometric signal measured. In such a configuration, the interfringe distance, or the length

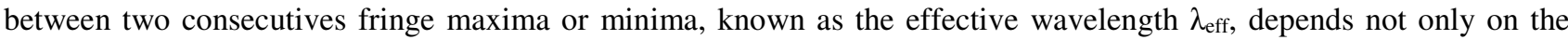
illumination wavelength $\lambda$, but also on the NA of the microscope objectives. Since it is difficult to theoretically calculate $\lambda_{\text {eff }}$ of the system with precision, this is normally measured experimentally from an interferogram after system calibration with a reference step. $\lambda_{\text {eff }}$ takes into account the whole response of the interferometric system and is important for accurate PSM measurements by introducing the correct phase shifts. 


\section{VERTICALLY ORIENTATED LINNIK INTERFEROMETER}

The first design, presented in Fig. 2, is an original vertically orientated breadboard interferometer in a Linnik configuration, and working in the reflective mode. A Linnik interferometer presents the distinctive feature of possessing identical microscope objectives in the reference and object arms, in order to be able to measure the sample topography with lateral- $\mu \mathrm{m}$-sized elements. The choice of components was made with the aim of reducing and minimizing the different sources of noise as much as possible.

First, Köhler illumination was chosen to optimally illuminate the sample. The main aims are to obtain a uniform and homogeneous sample illumination and identical spatial coherence at each of the sample points ${ }^{26}$. This enables the independent control of the illumination area on the sample and the angle of the illumination cone using respectively a field diaphragm (FD) and an aperture diaphragm (AD). A computer-assisted design with CODEV Optical Design Software was first made to dimension the illumination part of the system. After traveling through the lenses acting as Köhler illumination, the light from the white LED is divided into two beams with the same intensity for the reference arm and the object arm. In both arms, the light passes through identical high-quality long working distance apochromatic microscope objectives (Mitutoyo MY20X-804), corrected for infinity, with a x20 magnification, a NA equal to 0.42 and a working distance of $20 \mathrm{~mm}$. In the reference arm, the beam is reflected back by a mirror and in the object arm by the sample. These beams are then merged by travelling again through the beam splitter. Since the objectives are infinity corrected, a relay lens is necessary to produce the image on the camera sensor. For the image acquisition, a PCO Edge 4.2 camera with a scientific-CMOS (sCMOS) monochrome sensor interfaced with the computer in USB 3.0 is used. It also presents a higher dynamic range with the value encoded to $16 \mathrm{bit}$, and a better signal to noise ratio than with a classic CMOS sensor, while maintaining a high acquisition speed (170 fps for a 640 by 480 pixels image). Moreover, the camera is cooled with a Peltier system, which allows making measurements over a long period without drift.

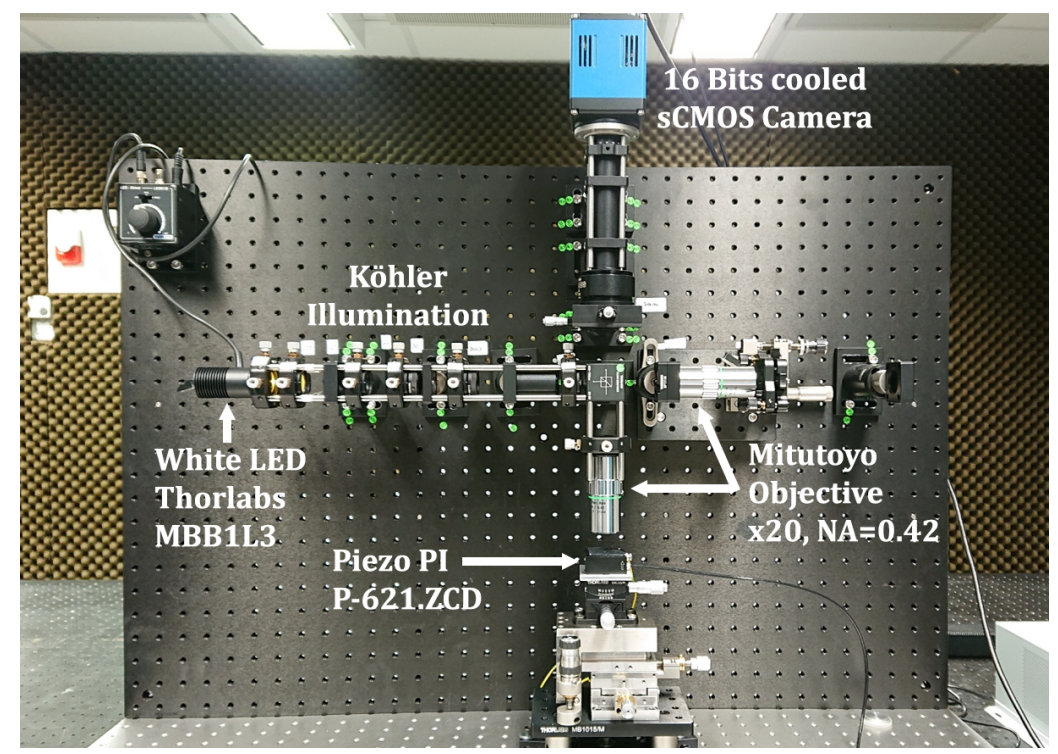

Figure 2. Vertically-orientated white light Linnik interferometer working in the reflective mode.

The sample is placed on a piezo electric translator (PI Hera P-621.ZCD), controlled in closed-loop allowing a position sensitivity along the optical axis of $+/-1 \mathrm{~nm}$. The piezo is placed on a 2-axis goniometer in order to adjust the inclination of the sample. Since a low coherence source is used, the fringe pattern appears only when the optical path difference between the object and reference arm is shorter than the coherence length of the source (several $\mu \mathrm{m}$ in this case). The whole of the reference arm is placed on a translation table with a $\mu \mathrm{m}$-displacement sensitivity to allow the independent control and alignment of the coherence plane and the focal plane positions for optimizing fringe contrast. The OPD in the Linnik interferometer also depends on the reference mirror tilt and from the distance separating the optical center of each microscope objective orthogonally to the system optical axis. To be able to optimize the fringe contrast and adjust the fringe spacing on an XY interferometric image by superimposing the coherence and focal planes, the mirror is mounted on a cage. This cage allows its inclination to be adjusted while the object arm objective is mounted on a translation cage. 
The goal of this setup is to be able to control the optical centers of the two objectives. A vertically orientated design was chosen to simplify the measurement of samples placed on the piezo system. The mechanical stability was reinforced by using low thermal coefficient materials and an optical table reinforced with struts, mounted on rubber vibration dampers.

The interferometric system is controlled by a home developed LabVIEW 2019 program using the IMAQVision imaging module (National Instruments). To increase the imaging contrast, the aperture diaphragm of the setup is closed. The effective wavelength measured experimentally with this system is equal to $\lambda_{\text {eff }}=660 \mathrm{~nm}$, and the lateral resolution obtained is about $\Delta \mathrm{x}=1000 \mathrm{~nm}$.

\section{COMPACT MIRAU INTERFEROMETER}

The second system developed is a compact white light interferometer based on a Mirau microscope objective (Fig. 3). The white light beam coming from the LED source is directly inserted into the beam splitter, which transfers half the light into the Nikon Mirau Objective (x10, NA=0.3). The objective is mounted on a piezo PI PIFOC P-721.C20, controlled in closed loop with a positioning sensitivity of $10 \mathrm{~nm}$, in order to modify the optical path difference. This objective is corrected for infinity, so the light reflected from the sample travels through a tube lens identical to that of the previous system. The image from the sample is recorded by an 8-bit high-speed color Basler camera (ACA640-750uc, about 800 frames per second for $640 * 860$ pixels, USB 3.0 connection).

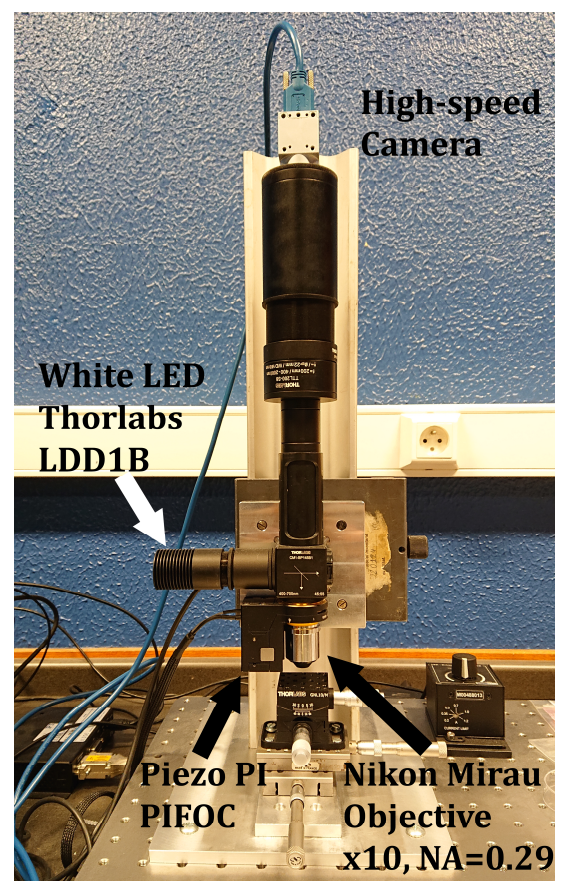

Figure 3. Home-made Mirau white light Interferometer.

The sample is placed on a goniometer (identical to the previous setup) to modify its orientation. The interferometer is directly included in the Mirau objective which is mounted at the precise position to give a magnification of 10x while respecting the right distance between the tube lens and the objective. The Mirau objective ensures the optimum superposition of the focal and coherence planes and guarantees a better mechanical stability than the Linnik configuration although it has less degrees of freedom. The optical setup is placed on an anti-vibration table with sorbothane isolators, acting as vibration absorbing feet. The acquisition (control of the camera and the piezo device) and the processing of the measurements are performed with IGORPro software or LabVIEW 2017. The effective wavelength measured through this system is equal to $\lambda_{\text {eff }}=580 \mathrm{~nm}$. Although difficult, since the fringe pattern always appears on the image of the sample, the lateral resolution $\Delta \mathrm{x}$, measured experimentally is about $1.2 \mu \mathrm{m}$. 


\section{SYSTEM PERFORMANCE}

\subsection{Interferometric measurement}

An example of a CSI measurement made with the Linnik system was carried out on a reference standard consisting of a square groove etched in quartz with a depth of $380 \mathrm{~nm}$. To obtain a good compromise between the measurement speed and the measurement accuracy, a piezo step of $25 \mathrm{~nm}$ was chosen over a dynamic range of $8 \mu \mathrm{m}$. At each step, 10 interferograms were averaged, to reduce the temporal noise. The resulting images were stored and then processed, with the previous algorithm described. An additional median ( 3 by 3 pixels) and averaging filter ( 2 by 2 pixels) were applied to further eliminate noise in the image. The 3D structure measured is illustrated in Fig 4(a), and a profile extracted in Fig 4(b). A typical height of $375 \mathrm{~nm}$ was found, but with heights varying between $350 \mathrm{~nm}$ and $400 \mathrm{~nm}$, due to the piezo step chosen which can only provide a sensitivity of $25 \mathrm{~nm}$, together with surface defects in the grating.

For the sake of comparison, a PSM measurement of the same standard was made with the Mirau setup described in section 4. The sample was axially moved 4 times, with successive piezo steps of $1 / 8$ of the effective wavelength, to introduce phase shifts of $\pi / 2$. As before, for each step, the interferogram stored was the average of 10 interferograms. The phase was obtained with the 5-step phase shifting algorithm, unwrapped, and converted into topographic information. The height obtained was then processed with identical median and averaging filters as before. The result obtained was smoother, as shown in Fig. 4(c), due to the method (PSM) used. An extracted profile is also presented in Fig. 4(d). The typical height measured is $379.1 \mathrm{~nm}$, with a standard deviation of $4.35 \mathrm{~nm}$. This results in an average error of $+/-1.15 \%$, with the standard deviation as a reference. It should be noted that similar results are obtained with both systems in PSM and CSI.

(a)

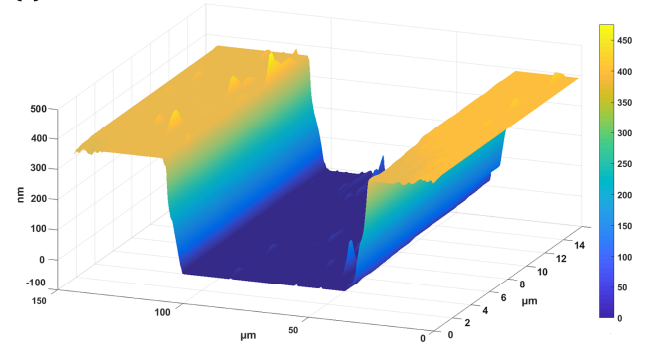

(c)

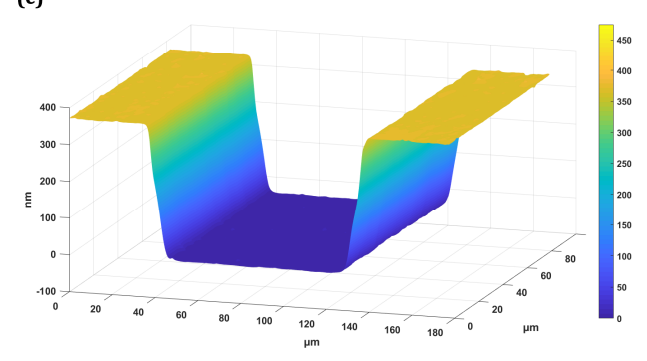

(b)

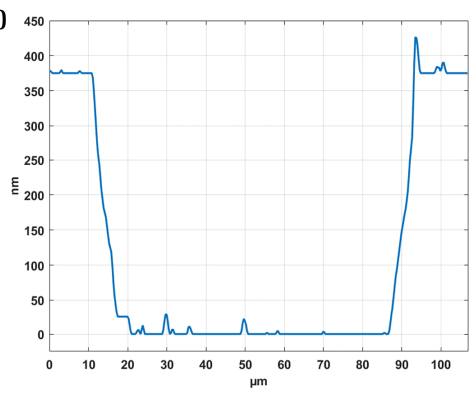

(d)

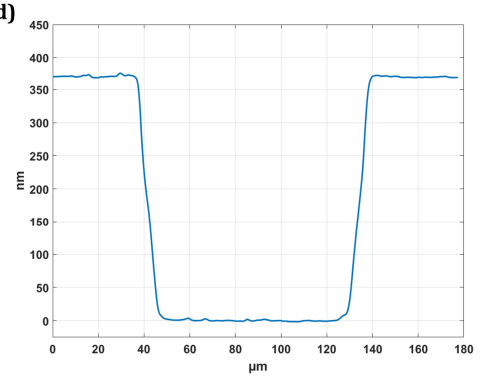

Figure 4. 3D results of a 380-nm step etched in quartz measured using PFSM with the Linnik configuration in (a), using PSM with the Mirau configuration in (c), together with extracted profiles respectively in (b) and in (d).

\subsection{Signal to Noise Ratio (SNR)}

An evaluation of the interferometric signal was performed, through the determination of different types of signal to noise ratio (SNR). The interferometric signal along each pixel was first processed, with the suppression of the offset, and then by taking its absolute value. The first signal to noise ratio definition (SNR1) is proposed in equation (1). SNR1 consists of the ratio of the mean value of the processed interferometric signal maximum $\left(\mu_{\max }\right)$, divided by the mean value of the noise amplitude at each pixel ( $\left.\mu_{\text {noise }}\right)$.

$$
S N R 1=10 . \log _{10}\left(\frac{\mu_{\max }}{\mu_{\text {noise }}}\right)
$$


A second SNR typically used in signal processing ${ }^{25}$, and named SNR2, is written in equation (2). SNR2 is the ratio of the mean square of the processed signal $R M S_{\text {signal }}^{2}$ on the variance of the noise $\sigma_{\text {noise }}^{2}$.

$$
S N R 2=10 . \log _{10}\left(\frac{R M S_{\text {signal }}^{2}}{\sigma_{\text {noise }}^{2}}\right)
$$

The value is determined for these 2 SNR with the Mirau system, the Linnik configuration and the Linnik Configuration with 10 images averaged for each piezo step. The results in $\mathrm{dB}$ is presented in Table 1. For SNR1 and SNR2, the values for the Linnik configuration (particularly for averaging 10 images) are better than those for the Mirau configuration, which can be explained by the low-noise 16-bit camera used with the Linnik system. If it is admitted that a value greater than $20 \mathrm{~dB}$ for SNR2 corresponds to high signal quality, the results demonstrates that a good interferometric signal is obtained for both setups.

Table 1. SNR value in dB determined for the Mirau, Linnik and Linnik (10 Avg) system. Linnik (10 Avg) consists in averaging 10 interferograms for each piezo step

\begin{tabular}{|c|c|c|c|}
\hline & Mirau & Linnik & Linnik (10 Avg) \\
\hline SNR1 & 14.96 & 19.56 & 23.61 \\
\hline SNR2 & 24.79 & 28.02 & 36.47 \\
\hline
\end{tabular}

\subsection{Lateral resolution of topographic system}

As mentioned in the introduction, the $3 \mathrm{D}$ response of the topographic measurement system should be considered to describe the performance of the optical setup. For this reason, the ability of both systems to measure the heights of gratings with different spatial frequencies was checked. To do so, a topographic reference standard (RS-N gratings from SiMETRICS Gmbh), made of a series of square profile gratings with different periods etched in a silicon wafer, allowed a rigorous study to be performed. Since the height of the gratings given by the manufacturer is $192 \mathrm{~nm}$, they were measured and processed with a 5-step phase shifting algorithm with 10 images averaged at each step. Due to the very straight edges of the square shaped grooves, at these positions a diffractive phenomenon, known as the batwing effect ${ }^{9,25}$, can appear in the phase. This can lead to misinterpretation by the $2 \mathrm{D}$ unwrapping algorithm, and so to wrong measurements of the topography, thus requiring special attention to processing. The measured height as a function of the period gratings $\Lambda$ is represented in Fig. 5(a), in red, blue and black respectively for the Mirau interferometer, Linnik interferometer and the characterization made by the manufacturer. In Fig. 5(b), the error E defined in equation (3), between the heights measured, $\mathrm{h}_{\text {meas }}$, and the height characterized by the manufacturer, $\mathrm{h}_{\mathrm{char}}$, is plotted with the same color code defined previously.

$$
E(\Lambda)=\frac{\left|h_{\text {char }}(\Lambda)-h_{\text {meas }}(\Lambda)\right|}{h_{\text {char }}(\Lambda)}
$$

To describe the performance of the systems, the 3D measurement sensitivities were determined. This was performed by measuring a flat silicon sample using PSM in order to determine the standard deviation of the local height distribution. The sensitivities were estimated as being $1.48 \mathrm{~nm}$, and $1.73 \mathrm{~nm}$ respectively for the Mirau and the Linnik interferometers. The errors between the measured groove heights and the data from the company, is not entirely explainable by these sensitivities. These errors could be for instance related to differences between the areas measured here and those measured by the manufacturer. For spatial frequencies smaller than $0.5 \mathrm{cycles} / \mu \mathrm{m}$, the error is negligible with the Linnik system. However, the errors in the heights measured observed for spatial frequency greater than 0.5 cycles $/ \mu \mathrm{m}$, cannot be explained only by the noise from the system. This error is rather due to the 3D optical transfer function of the system, i.e. taking into account the frequency response of the optical system but in 3 dimensions.

Indeed, the ability of the system to resolve lateral elements in 2D does not guarantee that reliable height information of these elements will be obtained. The 3D response of the interferometer does not depend only on its $2 \mathrm{D}$ imaging properties. This is why the lateral limit of measurable features with a 3D system is different, and usually lower, than the lateral resolution that can be achieved considering only its direct imaging capacities, as mentioned by Leach et al. ${ }^{23}$, De Groot et $a l .{ }^{24}$, and Lehmann et al. ${ }^{25}$. A 3D lateral limit needs to be established, by defining a criterion taking into 
consideration the aptitude of the system to perform relevant topographic information as a function of spatial frequency. For example, in Ref. ${ }^{24}$, a proposition is to define the 3D lateral limit as the spatial frequency from which the height measured is smaller than the half of the true height. Considering this proposition as a criterion, the green line is added in Fig. 5(b) corresponding to half of the height given by the manufacturer (and so to an error of $50 \%$ ). The intersection of this line (black dot), with the curve obtained with both system allows the definition of a 3D lateral limit of 0.547 cycles $/ \mu \mathrm{m}$ (i.e. $1.83 \mu \mathrm{m}$ ) and 0.658 cycles $/ \mu \mathrm{m}$ (i.e. $1.52 \mu \mathrm{m}$ ), compared to a 2D lateral resolution experimentally measured of $1.2 \mu \mathrm{m}$ and $1 \mu \mathrm{m}$, respectively for the Mirau and the Linnik interferometer. As expected, these results show a decrease in lateral resolution when considering the $3 \mathrm{D}$ response of the system, compared to its $2 \mathrm{D}$ response.

(a)

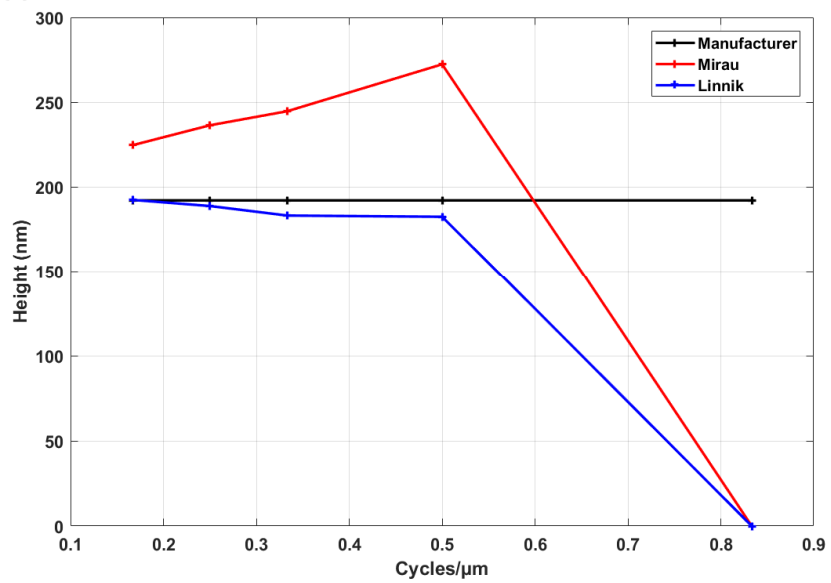

(b)

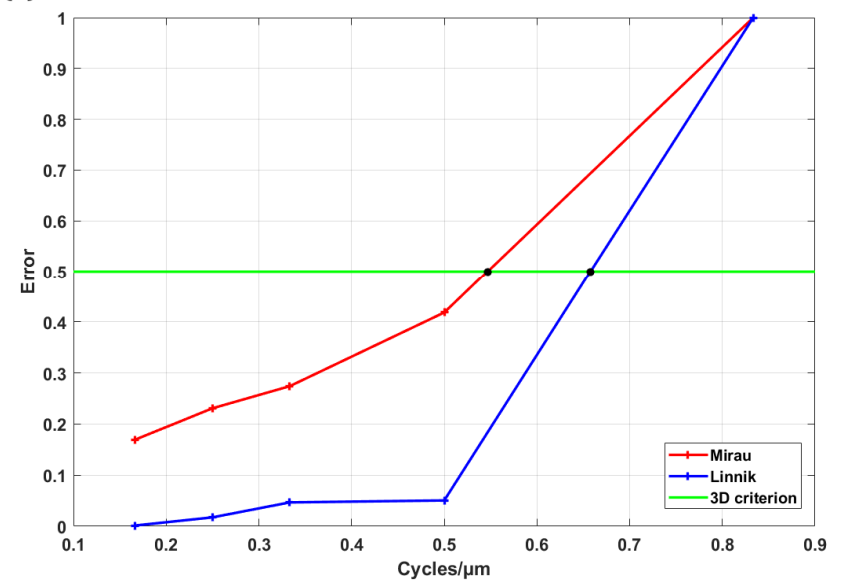

Figure 5. (a) Height measurement of the standards as a function of the period for the Mirau and Linnik interferometer respectively in red and blue compared with the value given by the manufacturer in black. (b) Error on the height measurement for both system with identical color code, in green the criterion for lateral limit of topographic system (error inferior to 50\%) and in black dots the intersection of the criterion with the system.

\section{CONCLUSION}

In this work, two original white light interferometers, working in the reflective mode, have been presented and experimentally studied. The first configuration is a vertical orientated breadboard Linnik interferometer. The components choice was based on the results of optical simulation, so as first to obtain experimentally a Köhler illumination and then to minimize the noise by using a 16-bit sCMOS cooled camera and a Piezo working in closed loop with nanometer sensitivity. A sufficient number of degrees of freedom was built in to allow the adjustment of necessary parameters without a too complicated alignment, and so as to give ease of modification. The second system developed was a compact Mirau Interferometer, which is easier to use than the Linnik, giving a high mechanical stability, but less degrees of freedom. The performance of each system was then determined, through classic interferometry measurement (PSM and CSI), and determination of signal to noise ratio values. Finally, the 3D lateral limit was compared to the 2D lateral resolution for both systems, in order to highlight the specificities of the interferometric systems, resulting in a difference in resolution as illustrated with the study conducted on spatial frequency response.

\section{REFERENCES}

[1] Conroy, M., and Armstrong, J., "A comparison of surface metrology techniques," J. Physics: Conference Series. 13(1), IOP Publishing, 458-465 (2005).

[2] Upadhyay, R. K., and Lakshmi A. K., "Bearing failure issues and corrective measures through surface engineering," in [Handbook of Materials Failure Analysis], Butterworth-Heinemann, 209-233 (2018).

[3] Tomovich, S., Peng, Z., Yuan, C., and Yan, X., "Quantitative surface characterisation using laser scanning confocal microscopy, " in [Laser Scanning, Theory and Applications], IntechOpen, 1-30 (2011). 
[4] De Groot, P., "Principles of interference microscopy for the measurement of surface topography," Adv. Opt. Photon. 7(1), 1-65 (2015).

[5] Niehues, J., Lehmann, P., and Xie, W. (2012), "Low coherent Linnik interferometer optimized for use in nanomeasuring machines," Meas. Sci. Technol. 23(12), 125002 (2012).

[6] Montgomery, P. C., Anstotz, F., Montagna, J., Montaner, D., Pramatarova, L., and Pecheva, E., "Towards real time 3D quantitative characterisation of in situ layer growth using white light interference microscopy," , J. Phys.: Conf. Ser. 253(1), 012017, IOP Publishing (2010).

[7] Wang, F., Liu, L., Yu, P., Liu, Z., Yu, H., Wang, Y., and Li, W. J., "Three-dimensional super-resolution morphology by near-field assisted white-light interferometry", Sci. Rep. 6(1), 1-10 (2016).

[8] Kassamakov, I., Lecler, S., Nolvi, A., Leong-Hoï, A., Montgomery, P., and Hæggström, E., "3D superresolution optical profiling using microsphere enhanced Mirau interferometry," Sci. Rep. 7(1), 1-7 (2017).

[9] Leong-Hoi, A., Hairaye, C., Perrin, S., Lecler, S., Pfeiffer, P., and Montgomery, P., "High Resolution Microsphere-Assisted Interference Microscopy for 3D Characterization of Nanomaterials," Phys. Status Solidi (a), 215(6), 1700858 (2018).

[10] Marbach, S., Perrin, S., Montgomery, P., Flury, M., and Lecler, S., "Microsphere-assisted imaging of subdiffraction-limited features", Proc. SPIE 11056, 110560R (2019).

[11]Coquoz, S., Nahas, A., Sison, M., Lopez, A., and Lasser, T., "High-speed phase-shifting common-path quantitative phase imaging with a piezoelectric actuator," J of Biomedical Optics 21(12), 126019 (2016).

[12] Montgomery, P., Anstotz, F., Johnson, G., and Kiefer, R., "Real time surface morphology analysis of semiconductor materials and devices using 4D interference microscopy," J. Mater. Sci. Mater. Electron. 19(1), 194-198 (2008).

[13] Coupland, J. M., and Lobera, J., "Measurement of Step Surfaces Using White Light Interferometry," Strain 46(1), 69-78 (2010).

[14] Montgomery, P. C., Montaner, D., Manzardo, O., Flury, M., and Herzig, H. P., "The metrology of a miniature FT spectrometer MOEMS device using white light scanning interference microscopy," Thin Solid Films 450(1), 79-83 (2004).

[15] Benatmane, A., and Montgomery, P. C., "3D analysis of buried interfaces using interference microscopy," Eur. Phys. J. App. Phys. 27(1-3), 411-414 (2004).

[16] Montgomery, P. C., Montaner, D., and Salzenstein, F., "Tomographic analysis of medium thickness transparent layers using white light scanning interferometry and XZ fringe image processing," Proc. SPIE 8430, 843014 (2012).

[17] Dubois, A., Vabre, L., Boccara, A.-C., and Beaurepaire, E., "High-Resolution Full-Field Optical Coherence Tomography with a Linnik Microscope," Appl. Opt. 41(4), 805-812 (2002).

[18] Dubois, A., Grieve, K., Moneron, G., Lecaque, R., Vabre, L., and Boccara, C., "Ultrahigh-resolution full-field optical coherence tomography," Appl. Opt. 43(14), 2874-2883 (2004).

[19] Nahas, A., Varna, M., Fort, E., and Boccara, A.-C., "Detection of plasmonic nanoparticles with full field-OCT: optical and photothermal detection," Biomed. Opt. Express 5(10), 3541-3546 (2014).

[20] Nahas, A., Bauer, M., Roux, S., and Boccara, A.-C., "3D static elastography at the micrometer scale using Full Field OCT," Biomed. Opt. Express 4(10), 2138-2149 (2013).

[21] Krauter, J., Boettcher, T., Körner, K., Gronle, M., Osten, W., Passilly, N., Froehly, L., Perrin, S., and Gorecki, C., "Full-field swept-source optical coherence tomography with phase-shifting techniques for skin cancer detection," Proc. SPIE 9529, 952913 (2015).

[22] Dubois, A., Levecq, O., Azimani, H., Siret, D., Barut, A., Suppa, M., Marmol, V., Malvehy, J., Cinotti, E., Rubegni, P., and Perrot, J. L. "Line-field confocal optical coherence tomography for high-resolution noninvasive imaging of skin tumors," J. of Biomedical Optics 23(10), 106007 (2018).

[23]Leach, R., Giusca, C., Henning, A., Sherlock, B., Coupland, J., "ISO Definition of Resolution for Surface Topography Measuring Instruments," in [Fringe 2013], eds Osten, W., Springer, 405-410 (2014).

[24] De Groot, P. and de Lega, X. C., "Interpreting interferometric height measurement using the instrument transfer function," in [Fringe 2015], eds Osten, W., Springer, 30-37 (2006).

[25]Lehmann, P., Tereschenko, S., and Xie, W., "Fundamental aspects of resolution and precision in vertical scanning white-light interferometry, " Surf. Topogr.: Metrol. Prop. 4(2), 024004 (2016).

[26]Köhler, A., "Ein neues Beleuchtungsverfahren für mikrophotographische Zwecke," Zeitschrift für wissenschaftliche Mikroskopie und für Mikroskopische Technik. 10 (4), 433-440 (1893). 